Original article

\title{
Functioning mediates help-seeking for mental problems in the general population
}

\author{
Chantal Michel ${ }^{\mathrm{a}, \mathrm{b}, *, 1}$, Nina Schnyder ${ }^{\mathrm{a}, 1}$, Stefanie J. Schmidt ${ }^{\mathrm{a}}$, Nicola Groth ${ }^{\mathrm{a}}$, \\ Benno G. Schimmelmann ${ }^{\mathrm{a}, \mathrm{c}}$, Frauke Schultze-Lutter ${ }^{\mathrm{a}, \mathrm{d}}$ \\ a University Hospital of Child and Adolescent Psychiatry and Psychotherapy, University of Bern, Bern, Switzerland \\ ${ }^{\mathrm{b}}$ Developmental Clinical Psychology Research Unit, Faculty of Psychology and Educational Sciences, University of Geneva, Switzerland \\ c University Hospital of Child and Adolescent Psychiatry, University Hospital Hamburg-Eppendorf, Hamburg, Germany \\ d Department of Psychiatry and Psychotherapy, Medical Faculty, Heinrich-Heine-University, Düsseldorf, Germany
}

\section{A R T I C L E I N F O}

\section{Article history:}

Received 17 May 2018

Received in revised form 27 June 2018

Accepted 27 June 2018

Available online 19 July 2018

\section{Keywords:}

Help-seeking

Axis-I problems/disorders

Functioning

Community

Path model

\begin{abstract}
A B S T R A C T
Aims: Absent or delayed help-seeking is considered to aggravate the immense personal and societal burden caused by mental disorders. Therefore, we cross-sectionally examined rates and clinical and sociodemographic moderators of early help-seeking for current clinician-assessed non-psychotic mental problems/disorders in the community.

Methods: Altogether, 2683 individuals of the Swiss Canton Bern (16-40 years old, response rate 63.4\%) were interviewed by telephone for current axis-I problems/disorders using the Mini-International Neuropsychiatric Interview, for psychosocial functioning using the Social and Occupational Functioning Assessment Scale, and for help-seeking for mental problems.

Results: In total, 1122 (41.8\%) reported mental problems. Of these, 769 (68.5\%) affirmed any one screening question and 353 (31.5\%) fulfilled criteria for any current axis-I disorder, and 396 (35.3\%) reported any lifetime help-seeking (28.3\% sought help in the past and $7.0 \%$ were in current treatment). In path analyses, current help-seeking was associated mainly by type and number of mental problems/disorders mediated by functional impairment, in addition to older age, no current partner, and past treatment. Conclusion: Our cross-sectional data indicate a gap in help-seeking for mental problems/disorders. The relationship between number of mental problems/disorders and help-seeking mediated by functional impairment confirm that individuals commonly do not seek help until problems are severe enough to cause problems in occupational and psychosocial functioning, driving the already immense costs of mental disorders. Thus, campaigns promoting early help-seeking, including early diagnostic clarification of and support for subthreshold mental problems in terms of an indicated prevention, should focus on psychosocial functioning, aside from signs of mental illness.
\end{abstract}

(c) 2018 Elsevier Masson SAS. All rights reserved.

\section{Introduction}

In 2010, approximately $38 \%$ of the European population suffered from a mental disorder [1]. Mental disorders commonly have their onset in late adolescence and early adulthood [2-4], and contributed most to the overall morbidity, already in 10- to 14year-olds [5], and annual costs in Europe in general [6] and Switzerland in particular [7].

\footnotetext{
* Corresponding author at: University Hospital of Child and Adolescent Psychiatry and Psychotherapy, Bolligenstrasse 111, Haus A, CH-3000 Bern 60, Switzerland.

E-mail address: chantal.michel@upd.unibe.ch (C. Michel).

1 Shared first authorship.
}

Absent or delayed help-seeking for mental problems, and the resulting high rates of chronic or recurrent courses are considered the main reasons for the high long-term burden caused by mental disorders [8]. Consequently, treatment for early incipient cases and an indicated prevention of mental illness, through the detection and treatment of early symptoms, are considered the most important aspects of both reducing severity-persistence of primary disorders and preventing secondary disorders and, thus, of reducing the overall burden [2-4,9]. Yet, despite increasing mental health service use in recent decades, the majority of individuals with mental problems do not seek professional help in terms of both diagnostic clarification and treatment [10-12]. Thus, the rate of the so called 'treatment gap' for people with mental problems exceeds 50\% around the world [13-16] (note: henceforth 'treatment gap' is used for the absence of any kind of help-seeking 
in persons with mental problems/disorders incl. seeking diagnostic clarification and temporary support).

To promote mental health by early help-seeking for mental problems in terms of both early treatment of manifest disorders and indicated prevention of incipient disorders, and to reduce long-term burden of mental disorders, a good understanding of detaining and promoting factors of help-seeking and their interplay is important. Among sociodemographic factors, previous studies found lower rates of help-seeking in men [17-20] and in younger age groups [21]. In contrast, obtaining a higher education, living alone, and being unemployed, disabled, or a single parent seemed to promote help-seeking [15,21,22]. An important clinical factor associated with more likely help-seeking is the presence of a manifest mental disorder, specifically a mood disorder [15,23], that commonly is leading to severe distress if not functional impairment. Previous studies, however, have not assessed the symptom-independent role of psychosocial functioning in helpseeking for mental disorders. In addition, they did not consider the role of satisfaction with possible earlier help-seeking attempts.

Thus, using semi-structured interviews conducted by clinical psychologists, we examined help-seeking for current mental disorders and problems possibly indicating an incipient disorder and, thus, a need for diagnostic clarification or mental health promotive actions. Moreover, advancing previous studies, we analysed the association of sociodemographic and clinical factors, including psychosocial functioning, and satisfaction with past or current treatment, to better understand the possible reasons for help-seeking.

\section{Material and methods}

\subsection{Sample}

The sample consisted of 2683 participants of the 'Bern Epidemiological At-Risk' (BEAR) study [24], a representative sample of the Bernese general population recruited using a stratified sampling method. Of the initial sample ( $\mathrm{N}=7370), 4471$ were considered eligible [24]. The contact rate was $94.8 \%$, the response rate $63.4 \%$ and the refusal rate $30.2 \%$.

In addition to being between 16 and 40 years-of-age and a main resident of Canton Bern (i.e. having a valid address and not being abroad during the assessment period), an available telephone number was required for eligibility [24]. We called participants up to 100 times over several months at various times and days, including Saturdays. Potential participants that were not reached within this time were considered as unknown eligible. Moreover, interviews were aborted prematurely when respondents had (i) a lifetime diagnosis of psychosis $(n=41)$ or (ii) insufficient language skills in German, French, or English $(\mathrm{n}=125)$. The former was done for the study's focus on clinical high risk for psychosis symptoms and criteria [24]. Of the 41 psychosis cases, 19 (46\%) had never been diagnosed and treated at the time of the interview (these cases are described in detail in [25]). Only eight participants prematurely aborted the interview on their own account. The semi-structured interviews lasted $43 \mathrm{~min}$ on average (SD: $20 \mathrm{~min}$; range: 20$225 \mathrm{~min}$ ).

The BEAR study was carried out in accordance with the latest version of the Declaration of Helsinki. The ethics committee of the University of Bern approved this study. Further details on recruitment and sample are provided in Schultze-Lutter et al. [24].

\subsection{Assessments}

\subsubsection{Axis-I disorders}

Present DSM-IV non-substance-related axis-I disorders were assessed using the Mini-International Neuropsychiatric Interview
(M.I.N.I.) [26], which was successfully applied in telephone surveys before [27], and was shown to be a reliable measure with good concurrent and predictive validity for assessing axis-I disorders [28] with satisfying reliability of telephone assessments compared with face-to-face assessments [29]. In line with the findings on the Modified Mini Screen (MMS) [30], which is based on the M.I.N.I., the presence of any subthreshold mental problem that signals a need of professional assessment and, consequently, a need for help-seeking was assumed when at least one screening question of the M.I.N.I. (eMaterial 1) was confirmed [30,31]. Yet, when diagnostic criteria of the respective disorder were additionally affirmed in the clinical interview, instead, we assumed presence of a current mental disorder. In an open question, we asked about any known neurological disorders to exclude mental problems/ disorders caused by them. Broadly in line with the M.I.N.I., current substance misuse (incl. dependence) was estimated predominately by frequency and quantity of consumption supplemented by questions on negative consequences or problems resulting from the substance use.

\subsubsection{Help-seeking}

Lifetime and current help-seeking as well as regular or sporadic current treatment for mental problems were assessed using a modified version of the WHO Pathway-to-Care questionnaire [32], which has previously been used successfully in international studies of help-seeking for mental disorders [32-34]. Except when stated otherwise, 'any help-seeking contact' included any reported point-of-call to a person other than a family member or friend, irrespective of his/her professional background. When a distinction was made, professional mental healthcare was assumed when provided by psychiatrists, psychologists, and counselling services staffed with psychologists, while semi-professional mental healthcare was assumed when provided by general practitioners or other medical specialists. Only when provided by (semi-)professionals, the term 'treatment' was used.

'Satisfaction with the effectiveness of treatment' (henceforth: treatment satisfaction) was assessed using an optional item of the Brief Multidimensional Life Satisfaction Scale (BLMSS) [35], which was rated on a seven-point Likert scale, ranging from 'horrible' $=0$ to 'very happy' $=6$. The BLMSS has good psychometric properties and can be regarded as a brief, reliable and valid measure of life satisfaction [35].

\subsubsection{Psychosocial functioning}

The level of psychosocial functioning was estimated using the Social and Occupational Functioning Assessment Scale (SOFAS), a rating scale for Axis- $\mathrm{V}$, the clinician's judgment of overall level of functioning, of DSM-IV [36]. The SOFAS is a global rating of current functioning ranging from 0 to 100 , with lower scores representing lower functioning, and is focusing on functioning independent of the overall severity of the individual's psychological symptoms [36]. It has good psychometric properties incl. good interraterreliability and construct validity $[37,38]$. A SOFAS-score between 31 and 70 refers to manifest disabilities of various degrees and a score below 30 reflects poor functioning making intensive support or supervision necessary [39]. Therefore, a score of $\leq 70$ was regarded as indicative of a presence of a functional deficit [39,40].

\subsubsection{Quality assurance}

To achieve a $\geq 95 \%$ concordance rate with the trainers (F.S.-L. and C.M.), interviewers (all clinical psychologists) received intensive 3-month training, especially in the semi-structured, context-dependent assessment of mental disorders. Additionally, weekly supervision of all psychopathological ratings with the interviewers was performed by the trainers to further ensure excellent, valid and reliable data quality. 


\subsection{Statistical analyses}

Group comparisons of categorical, or non-normally distributed continuous or ordinal data were assessed using $\chi^{2}$-tests or MannWhitney $U$ test Associations of sociodemographic and clinical variables with help-seeking were explored using univariate logistic regressions. Their goodness-of-fit was estimated using the Omnibus test. According to these results and theoretical considerations, path models were computed with the weighted least squares and variance adjusted estimator [41] based on diagonally weighted least squares for categorical variables [42]. Missing data were listwise deleted. The model fit was assessed using five commonly used indices, namely, the $\chi^{2}$ test, comparative fit index (CFI), Tucker-Lewis index (TLI), standardized root mean square residual (SRMR), and root-mean-square error of approximation (RMSEA), including a 90\%-confidence interval. We first tested the proposed model. After removing insignificant associations and receiving a final model, we performed sensitivity analyses of the final model for genders separately. Statistical analyses were conducted in SPSS 24.0 and R (R Core Team) package lavaan [43].

\section{Results}

Lack of time or interest was the main reason given by the refusers who differed marginally from the 2683 interviewees in age, sex, and Swiss nationality; yet, all differences were of extremely small effect size $(<0.040)$ [24]. Interviewees differed negligibly from the 16- to 40 -year-old general population of Bern in only age distribution, but not in gender, nationality, and marital status. Consequently, with not even a small-sized response bias being detectable, interviewees were regarded as representative of their local age group [24].

\subsection{Prevalence of current mental disorders/problems}

Of the 2683 interviewees, 1122 (41.8\%) individuals affirmed any M.I.N.I. screening question for non-psychotic axis-I disorders. Of these, 769 (28.7\%) only had subthreshold mental problems, and 353 (13.2\%) fulfilled criteria for any current axis-I disorder, including the most frequent specific phobias (6.5\%) (Table 1). Sixty-six (2.5\%) interviewees met criteria for more than one disorder; specific phobia was the most frequent comorbid disorder $(\mathrm{n}=22,0.8 \%)$.

Of the 1122 individuals with a mental problem/disorder, 41 (3.6\%) were additionally considered to currently misuse a substance; 19 (1.7\%) alcohol; 22 (2.0\%) psychotropic drugs or medication; and $4(0.1 \%)$ both.

\subsection{Prevalence of and reasons for help-seeking}

Six-hundred fifteen (22.9\%) interviewees reported any lifetime help-seeking for mental problems, and 95 (3.5\%) were currently in any contact for mental health problems. Of these current helpseekers, 30 (31.6\%) were in sporadic contact with a (semi-) professional at the time of the interview, and $49(51.5 \%)$ were in regular treatment. Thirty (31.6\%) of the 95 current help-seekers reported subthreshold mental problems; 48 (50.5\%) any mental disorder; and 17 (17.9\%) neither problems nor disorders.

Of the 1122 participants with any current mental problem/ disorder, 396 (35.3\%) reported having sought help at any time; $28.3 \%$ did so in the past and $7 \%$ were currently help-seeking (Table 2). Regarding 'satisfaction with the effectiveness of treatment', no difference was found between those with only past help-seeking and those with current help-seeking $(4.42 \pm 1.5$ vs. $4.65 \pm 1.3 ; \mathrm{U}=7466.0, \mathrm{p}=0.425)$. Of the 769 participants with subthreshold mental problems, $224(29.1 \%)$ reported having sought help for a mental problem at any time; $25.2 \%$ of the 769 did so in the past and $9 \%$, currently. Again, no difference in 'treatment satisfaction' was found between past and current helpseekers ( $4.55 \pm 1.5$ vs. $4.91 \pm 1.3 ; \mathrm{U}=1315.5, \mathrm{p}=0.254)$. Of the 353 participants with any current axis-I disorder, 172 (48.7\%) reported having sought help for a mental problem at any time; $35.1 \%$ of the 353 reported having sought help in the past and $13.6 \%$ reported current help-seeking. 'Treatment satisfaction' again did not differ between past and current help-seekers ( $4.25 \pm 1.5$ vs. $4.51 \pm 1.6$; $\mathrm{U}=2074.5, \mathrm{p}=0.427$ ).

With less than 30\%, the rate for any current help-seeking contacts was low for all non-psychotic axis-I problems/disorders (Table 2). Regarding (semi-)professional treatment, $12.7 \%$ of those with an affective, $6.2 \%$ of those with an anxiety, $7.8 \%$ of those with an eating, $9.9 \%$ of those with a somatoform, $11.9 \%$ of those with an obsessive-compulsive, and $8.7 \%$ of those with a post-traumatic stress problem/disorder were in treatment and received medication and/or psychotherapy.

\subsection{Moderators of help-seeking}

\subsubsection{Gender}

Women reported more often any lifetime help-seeking for a mental problem than men ( $31.5 \%$ vs. $15.6 \%$ ). However, women with a current full-blown disorder had lower rates of current helpseeking than affected men (16.7\% vs. $11.6 \%$ ) (Table 3$)$. When sporadic and regular current treatments were distinguished, no gender differences between men and women with any mental problem/disorder $\left(\chi_{(1)}^{2}=3.106, p=0.078\right)$, subthreshold mental problems $\left(\chi_{(1)}^{2}=2.738, p=0.098\right)$, and any current axis-I disorders $\left(\chi_{(1)}^{2}=0.820, \mathrm{p}=0.365\right)$ were detected.

\subsubsection{Functioning}

Only 147 interviewees (5.5\%) had a functional deficit, i.e. a SOFAS score below 71. Of these 147 persons, the majority ( $n=75$; $51.1 \%$ ) was not help-seeking although they had a current axis-I disorder, 31 (21.1\%) were currently help-seeking and had a current axis-I disorder, 6 persons $(4.1 \%)$ were currently help-seeking although they had no current axis-I disorder, and 35 participants (23.8\%) neither were help-seeking nor had a current axis-I disorder. Further, of the 353 persons with a current axis-I disorder, only $17(4.8 \%)$ without a functional deficit were currently helpseeking, while the majority of those with a current axis-I disorder $(n=230 ; 65.2 \%)$ did not seek help and did not have a SOFAS score below 71 . No gender difference in functional deficits were detected $\left(\chi_{(1)}^{2}=0.789, \mathrm{p}=0.374\right.$, Cramer's V=0.047).

\subsubsection{Other moderators}

Of the other sociodemographic variables, no current partner and a family history of mental disorders in first- or second-degree biological relatives (specifically of affective disorder) were predictive of current help-seeking in the 1122 participants with any current mental problem/disorder. Of the clinical variables, current drug misuse, a higher number of mental problems/ disorders, any current affective problem/disorder, and a current functional deficit predicted any current help-seeking (Table 4).

\subsubsection{Path model}

The proposed path model of the interplay of different sociodemographic and clinical factors on any current help-seeking, which was based on earlier models and the results of the univariate analyses, is reported in eFig. 1. This initial model had a good model fit (eFig. 2) that improved further after the elimination of five insignificant paths (Fig. 1). In this final model, the effect from number of problems/disorders on any current help-seeking was mediated significantly by functional deficit with a total effect of 
Table 1

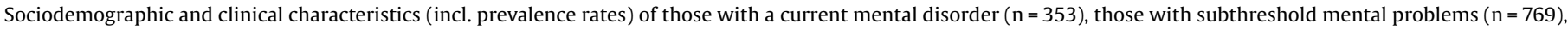
those with a current mental disorder and/or problem $(n=1122)$, those with neither a disorder nor a problem $(n=1561)$ and the total sample $(\mathrm{N}=2683)$.

\begin{tabular}{|c|c|c|c|c|c|c|c|c|c|c|}
\hline & \multicolumn{2}{|l|}{$\begin{array}{l}\text { Mental disorder } \\
\mathrm{n}=353\left(13.2 \%^{\mathrm{a}}\right)\end{array}$} & \multicolumn{2}{|c|}{$\begin{array}{l}\text { Subthreshold mental } \\
\text { problem } \\
n=769\left(28.7 \%^{\mathrm{a}}\right)\end{array}$} & \multicolumn{2}{|c|}{$\begin{array}{l}\text { Either } \\
\text { (disorder or problem) } \\
\mathrm{n}=1,122\left(41.8 \%^{\mathrm{a}}\right)\end{array}$} & \multicolumn{2}{|l|}{$\begin{array}{l}\text { Neither } \\
\text { (no disorder, no } \\
\text { problem) } \\
n=1,561\left(58.2 \%^{\mathrm{a}}\right)\end{array}$} & \multicolumn{2}{|l|}{$\begin{array}{l}\text { Total sample } \\
\mathrm{N}=2,683(100 \%)\end{array}$} \\
\hline & $\mathrm{n}$ & $\%$ & $\mathrm{n}$ & $\%$ & $\mathrm{n}$ & $\%$ & $\mathrm{n}$ & $\%$ & $\mathrm{~N}$ & $\%$ \\
\hline Age $($ mean \pm SD) & $30.8 \pm 7.6$ & & $30.5 \pm 7.5$ & & $30.6 \pm 7.5$ & & $30.7 \pm 7.6$ & & $30.7 \pm 7.6$ & \\
\hline Gender (\% male) & 144 & 40.8 & 351 & 45.6 & 495 & 44.1 & 952 & 61 & 1447 & 53.9 \\
\hline Nationality (\% Swiss) & 326 & 92.4 & 714 & 92.8 & 1040 & 92.7 & 1472 & 94.3 & 2512 & 93.6 \\
\hline Current partnership (\% yes) & 230 & 65.2 & 575 & 74.8 & 805 & 71.7 & 1131 & 72.5 & 1936 & 72.2 \\
\hline \multicolumn{11}{|l|}{ Highest education (\% yes) } \\
\hline $\begin{array}{l}\text { Primary school or school for special needs } \\
\text { (6 school years) }\end{array}$ & 4 & 1.1 & 11 & 1.4 & 15 & 1.3 & 20 & 1.3 & 35 & 1.3 \\
\hline Secondary school (9-10 school years) & 218 & 61.8 & 456 & 59.3 & 674 & 60.1 & 982 & 59.5 & 1602 & 59.7 \\
\hline High school (12-13 school years) & 131 & 37.1 & 302 & 39.3 & 433 & 38.6 & 613 & 39.3 & 1046 & 39 \\
\hline Current employment (\%employed) ${ }^{\mathrm{b}}$ & 332 & 94.3 & 748 & 97.3 & 1080 & 96.3 & 1539 & 98.7 & 2619 & 97.7 \\
\hline Family historyc (\% yes) & 177 & 50.1 & 324 & 42.1 & 501 & 44.7 & 524 & 33.6 & 1025 & 38.2 \\
\hline SOFAS score (mean \pm SD, median, range) & $\begin{array}{l}76.2 \pm 11.6,80.0 \\
39-95\end{array}$ & & $\begin{array}{l}85.3 \pm 5.6,86.0 \\
55-95\end{array}$ & & $\begin{array}{l}82.4 \pm 9.0,85.0 \\
39-95\end{array}$ & & $\begin{array}{l}87.4 \pm 4.1,88.0 \\
60-99\end{array}$ & & $\begin{array}{l}85.3 \pm 7.1,87.0 \\
39-99\end{array}$ & \\
\hline Functional deficit ${ }^{\mathrm{d}}$ (\% yes) & 106 & 30 & 25 & 3.3 & 131 & 11.7 & 16 & 1 & 147 & 5.5 \\
\hline Current alcohol misuse (\%yes) & 9 & 2.5 & 10 & 1.3 & 19 & 1.7 & 15 & 1 & 34 & 1.3 \\
\hline Current drug misuse (\% yes) & 10 & 2.8 & 12 & 1.6 & 22 & 2 & 19 & 1.2 & 41 & 1.5 \\
\hline $\begin{array}{l}\text { Number axis-I disorders/problems } \\
\text { (mean } \pm \mathrm{SD} \text {, median, range) }\end{array}$ & $\begin{array}{l}1.80 \pm 1.0,2.0 \\
0-5\end{array}$ & & $\begin{array}{l}1.28 \pm 0.637 \\
1.0,0-4\end{array}$ & & $\begin{array}{l}1.45 \pm 0.81,1.0 \\
0-5\end{array}$ & & n.a. & & see left columns & \\
\hline Any affective disorder/problem (\% yes) & 112 & $4.2^{\mathrm{a}}$ & 208 & $7.8^{\mathrm{a}}$ & 362 & $13.5^{\mathrm{a}}$ & & & & \\
\hline Dysthymia & 50 & $1.9^{\mathrm{a}}$ & 51 & $1.9^{\mathrm{a}}$ & 135 & $5.0^{\mathrm{a}}$ & & & & \\
\hline Major depression & 76 & $2.8^{\mathrm{a}}$ & 131 & $4.9^{\mathrm{a}}$ & 237 & $8.8^{\mathrm{a}}$ & & & & \\
\hline Manic episode & 10 & $0.4^{\mathrm{a}}$ & 47 & $1.8^{\mathrm{a}}$ & 78 & $2.9^{\mathrm{a}}$ & & & & \\
\hline Any anxiety disorder/problem (\% yes) & 252 & $9.4^{\mathrm{a}}$ & 450 & $16.8^{\mathrm{a}}$ & 742 & $27.7^{\mathrm{a}}$ & & & & \\
\hline Generalized anxiety disorder & 36 & $1.3^{\mathrm{a}}$ & 69 & $2.6^{\mathrm{a}}$ & 148 & $5.5^{\mathrm{a}}$ & & & & \\
\hline Panic disorder & 26 & $1.0^{\mathrm{a}}$ & 76 & $2.8^{\mathrm{a}}$ & 127 & $4.7^{\mathrm{a}}$ & & & & \\
\hline Agoraphobia & 34 & $1.3^{\mathrm{a}}$ & 69 & $2.6^{\mathrm{a}}$ & 136 & $5.1^{\mathrm{a}}$ & & & & \\
\hline Social phobia & 22 & $0.8^{\mathrm{a}}$ & 66 & $2.5^{\mathrm{a}}$ & 117 & $4.4^{\mathrm{a}}$ & & & & \\
\hline Specific phobia & 174 & $6.5^{\mathrm{a}}$ & 278 & $10.4^{\mathrm{a}}$ & 477 & $17.8^{\mathrm{a}}$ & & & & \\
\hline Post-traumatic stress disorder & 19 & $0.7^{\mathrm{a}}$ & 212 & $7.9^{\mathrm{a}}$ & 283 & $10.5^{\mathrm{a}}$ & & & & \\
\hline Obsessive compulsive disorder & 21 & $0.8^{\mathrm{a}}$ & 40 & $1.5^{\mathrm{a}}$ & 84 & $3.1^{\mathrm{a}}$ & & & & \\
\hline Any eating disorder/problem (\% yes) & 12 & $0.4^{\mathrm{a}}$ & 31 & $1.2^{\mathrm{a}}$ & 64 & $2.4^{\mathrm{a}}$ & & & & \\
\hline Anorexia nervosa & 3 & $0.1^{\mathrm{a}}$ & 9 & $0.3^{\mathrm{a}}$ & 18 & $0.7^{\mathrm{a}}$ & & & & \\
\hline Bulimia nervosa & 10 & $0.4^{\mathrm{a}}$ & 23 & $0.9^{\mathrm{a}}$ & 49 & $1.8^{\mathrm{a}}$ & & & & \\
\hline Any somatoform disorder/problem (\% yes) & 27 & $1.0^{\mathrm{a}}$ & 84 & $3.1^{\mathrm{a}}$ & 161 & $6.0^{\mathrm{a}}$ & & & & \\
\hline Somatization disorder & 7 & $0.3^{\mathrm{a}}$ & 25 & $0.9^{\mathrm{a}}$ & 50 & $1.9^{\mathrm{a}}$ & & & & \\
\hline Hypochondriasis & 5 & $0.2^{\mathrm{a}}$ & 21 & $0.8^{\mathrm{a}}$ & 42 & $1.6^{\mathrm{a}}$ & & & & \\
\hline Body dysmorphic disorder & 7 & $0.3^{\mathrm{a}}$ & 25 & $0.9^{\mathrm{a}}$ & 48 & $1.8^{\mathrm{a}}$ & & & & \\
\hline Pain disorder & 10 & $0.4^{\mathrm{a}}$ & 28 & $1.0^{\mathrm{a}}$ & 57 & $2.1^{\mathrm{a}}$ & & & & \\
\hline
\end{tabular}

a All prevalences refer to the total sample.

b Includes sheltered employment, temporary employment, and regular full- and part-time employment (incl. schooling, academic studies, occupational training, full-time house work).

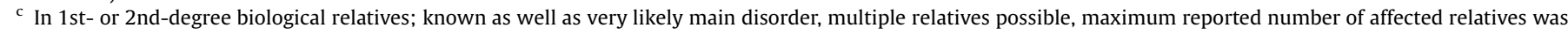
$\mathrm{n}=5$.

d Defined by a SOFAS score of 70 or less.Source: Bern Epidemiological At Risk (BEAR) Study (SNF project number: 135381). 
Table 2

Distribution of any current help-seeking for various current axis-I disorders and subthreshold problems $(n=1122)$.

\begin{tabular}{|c|c|c|}
\hline Type of disorder/problem & Currently in any treatment (\%) & Currently not in treatment (\%) \\
\hline Any disorder/problem $(n=1122)$ & 7.0 & 93.0 \\
\hline Depressive episode $(n=237)$ & 16.5 & 83.5 \\
\hline Dysthymia $(\mathrm{n}=135)$ & 17.0 & 83.0 \\
\hline Manic episode $(n=78)$ & 12.8 & 87.2 \\
\hline Generalized anxiety disorder $(n=148)$ & 20.3 & 79.7 \\
\hline Panic disorder $(n=127)$ & 13.4 & 86.6 \\
\hline Agoraphobia $(n=136)$ & 9.5 & 90.5 \\
\hline Social phobia $(n=117)$ & 6.8 & 93.2 \\
\hline Specific phobia $(n=477)$ & 4.0 & 96.0 \\
\hline Post-traumatic stress disorder $(n=283)$ & 7.1 & 92.9 \\
\hline Obsessive compulsive disorder $(n=84)$ & 13.1 & 86.9 \\
\hline Anorexia nervosa $(\mathrm{n}=18)$ & 11.5 & 89.0 \\
\hline Bulimia nervosa $(n=49)$ & 10.2 & 89.8 \\
\hline Somatization disorder $(n=50)$ & 14.0 & 86.0 \\
\hline Hypochondriasis $(n=42)$ & 9.5 & 90.5 \\
\hline Body dysmorphic disorder $(n=48)$ & 23.9 & 79.1 \\
\hline Pain disorder $(n=57)$ & 12.3 & 87.7 \\
\hline
\end{tabular}

Source: Bern Epidemiological At Risk (BEAR) Study (SNF project number: 135381).

Table 3

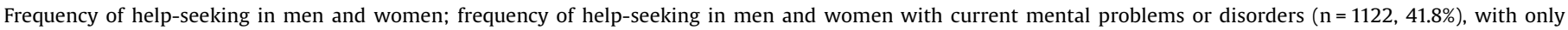
subthreshold current mental problems $(n=769,28.7 \%)$, and with any current axis-I disorder $(n=353,13.2 \%)$.

\begin{tabular}{|c|c|c|c|}
\hline & $\begin{array}{l}\text { Men } \\
1,447(53.9) \\
\end{array}$ & $\begin{array}{l}\text { Women } \\
1,236(46.1)\end{array}$ & Statistics \\
\hline $\begin{array}{l}\text { Help-seeking }(\mathrm{N}=2683) \\
\text { Never had a help-seeking contact } \\
\text { Help-seeking contact in the past } \\
\text { Any current help-seeking contact }\end{array}$ & $\begin{array}{l}226(15.6)^{\mathrm{a}} \\
1221(84.4)^{\mathrm{a}^{*}} \\
185(12.8)^{\mathrm{a}^{*}} \\
41(2.8)^{\mathrm{a}}\end{array}$ & $\begin{array}{l}389(31.5)^{\mathrm{c}} \\
847(68.5)^{\mathrm{c}^{*}} \\
335(27.1)^{\mathrm{c}^{*}} \\
54(4.4)^{\mathrm{c}}\end{array}$ & $\begin{array}{l}\chi_{(1)}^{2}=94.887, p<0.001, \text { Cramer's } V=0.188 \\
\chi_{(2)}^{2}=96.691, p<0.001, \text { Cramer's } V=0.190\end{array}$ \\
\hline $\begin{array}{l}\text { With current mental problems or disorders }(\mathrm{n}=1122) \\
\text { Never had a help-seeking contact } \\
\text { Help-seeking contact in the past } \\
\text { Any current help-seeking contact }\end{array}$ & $\begin{array}{l}495(34.2)^{\mathrm{a}} \\
355(71.7)^{\mathrm{b}} \\
103(20.8)^{\mathrm{b}^{*}} \\
37(7.5)^{\mathrm{b}}\end{array}$ & $\begin{array}{l}627(50.7)^{\mathrm{c}} \\
371(59.2)^{\mathrm{d}} \\
215(34.3)^{\mathrm{d}^{*}} \\
41(6.5)^{\mathrm{d}}\end{array}$ & $\begin{array}{l}\chi_{(1)}^{2}=75.097, p<0.001, \text { Cramer's } V=0.167 \\
\chi_{(2)}^{2}=24.818, p<0.001, \text { Cramer's } V=0.149\end{array}$ \\
\hline $\begin{array}{l}\text { With only subthreshold current mental problems }(\mathrm{n}=769) \\
\text { Never had a help-seeking contact } \\
\text { Help-seeking contact in the past } \\
\text { Any current help-seeking contact }\end{array}$ & $\begin{array}{l}351(24.3)^{\mathrm{a}} \\
275(78.3)^{\mathrm{e}} \\
63(17.9)^{\mathrm{e}^{*}} \\
13(3.7)^{\mathrm{e}}\end{array}$ & $\begin{array}{l}418(33.8)^{\mathrm{c}} \\
270(64.3)^{\mathrm{f}} \\
131(31.3)^{\mathrm{f}^{*}} \\
17(4.0)^{\mathrm{f}}\end{array}$ & $\begin{array}{l}\chi_{(1)}^{2}=29.806, p<0.001, \text { Cramer's } V=0.105 \\
\chi_{(2)}^{2}=18.719, p<0.001, \text { Cramer's } V=0.156\end{array}$ \\
\hline $\begin{array}{l}\text { With any current axis-I disorder }(\mathrm{n}=353) \\
\text { Never had a help-seeking contact } \\
\text { Help-seeking contact in the past } \\
\text { Any current help-seeking contact }\end{array}$ & $\begin{array}{l}144(10.0)^{\mathrm{b}} \\
80(55.6)^{\mathrm{g}} \\
40(27.8)^{\mathrm{g}} \\
24(16.7)^{\mathrm{g}}\end{array}$ & $\begin{array}{l}209(16.9)^{\mathrm{d}} \\
101(48.8)^{\mathrm{h}} \\
84(40.1)^{\mathrm{h}} \\
24(11.6)^{\mathrm{h}}\end{array}$ & $\begin{array}{l}\chi_{(1)}^{2}=28.243, p<0.001, \text { Cramer's } V=0.103 \\
\chi_{(2)}^{2}=6.294, p=0.043, \text { Cramer's V }=0.134\end{array}$ \\
\hline
\end{tabular}

a Percentages relate to all men.

b Percentages relate to men with mental problems or disorder $(n=495)$.

c Percentages relate to all women.

d Percentages relate to women with mental problems or disorder $(n=627)$.

e Percentages relate to men with only mental problems $(n=351)$.

f Percentages relate to women with only mental problems $(n=418)$.

g Percentages relate to men with mental disorder $(n=144)$.

h Percentages relate to women with mental disorder $(n=209)$.

Cell frequency significantly higher or lower than expected with the standardized residuum of cell of $>1.96$ and of $\leq 1.96$, respectively.Source: Bern Epidemiological At Risk (BEAR) Study (SNF project number: 135381).

$\mathrm{r}=0.317(\mathrm{p} \leq 0.001)$ and an indirect effect of $\mathrm{r}=0.288(\mathrm{p} \leq 0.001)$. The paths from number of problems/disorders to current functional deficit $(r=0.564, p \leq 0.001)$ and from current functional deficit to any current help-seeking $(r=0.511, p \leq 0.001)$ were significant, while the direct path from number of problems/ disorders to any current help-seeking was insignificant $(r=0.028$, $\mathrm{p}=0.672$ ).

The sensitivity analyses of the influence of gender revealed two slightly different models, specifically with regard to the role of age, of no current partner and past treatment (eFigs. 3 and 4). In females, past treatment was not associated with current helpseeking; whereas in males, age and no current partner were not associated with current help-seeking. Compared to the final model of the full sample, model fits in the subsamples declined and, in case of the female model, did not reach sufficient fit indices (eFigs. 3 and 4).

\section{Discussion}

This study's comprehensive model of predictors of current help-seeking for mental problems demonstrated the importance of deficits in psychosocial functioning as a mediator of the effect of mental problems/disorders, indicating that help is mostly only sought when mental problems are already so severe or many that they impair functioning. In light of indicated-preventive efforts this is unfortunate because, generally, these target not only the prevention of a manifest disorder but also of accompanying functional deficits that, once having manifested themselves, are 
Table 4

Potential predictors of any current help-seeking in those with any current mental problem or disorder $(\mathrm{N}=1122)$. Results of univariate logistic regression analyses.

\begin{tabular}{|c|c|c|c|c|c|c|c|c|}
\hline & $\beta$ & SE & Wald & df & p-value & $\operatorname{Exp}(\beta)$ & lower & $\begin{array}{l}\text { of } \operatorname{Exp}(\beta) \\
\text { upper }\end{array}$ \\
\hline $\mathrm{Age}^{\mathrm{a}}$ & 0.032 & 0.017 & 3.703 & 1 & 0.054 & 1.032 & 0.999 & 1.066 \\
\hline Gender $(\text { male })^{b}$ & 0.144 & 0.235 & 0.374 & 1 & 0.541 & 1.155 & 0.728 & 1.831 \\
\hline School education ${ }^{b}$ & 0.022 & 0.156 & 0.019 & 1 & 0.889 & 1.022 & 0.752 & 1.388 \\
\hline Current unemployment ${ }^{b}$ & 0.057 & 0.612 & 0.009 & 1 & 0.925 & 1.059 & 0.319 & 3.511 \\
\hline Migrant status ${ }^{b}$ & -0.401 & 0.527 & 0.581 & 1 & 0.446 & 0.669 & 0.238 & 1.879 \\
\hline Single marital status ${ }^{b}$ & 0.275 & 0.244 & 1.266 & 1 & 0.260 & 1.316 & 0.816 & 2.124 \\
\hline No current partner ${ }^{\mathrm{a}}$ & 0.845 & 0.238 & 12.640 & 1 & $<0.001$ & 2.327 & 1.461 & 3.708 \\
\hline $\begin{array}{l}\text { Positive family history of mental } \\
\text { disorders }\end{array}$ & 0.613 & 0.239 & 6.594 & 1 & 0.010 & 1.845 & 1.156 & 2.945 \\
\hline Current alcohol misuse ${ }^{b}$ & 0.944 & 0.640 & 2.172 & 1 & 0.141 & 2.570 & 0.732 & 9.017 \\
\hline Current drug misuse ${ }^{\mathrm{b}}$ & 1.125 & 0.566 & 3.957 & 1 & 0.047 & 3.081 & 1.017 & 9.338 \\
\hline Current functional deficit ${ }^{3}$ & 2.147 & 0.251 & 73.000 & 1 & $<0.001$ & 8.562 & 5.232 & 14.013 \\
\hline $\begin{array}{l}\text { Number of mental } \\
\text { problems/disorders }\end{array}$ & 0.634 & 0.114 & 30.783 & 1 & $<0.001$ & 1.886 & 1.507 & 2.360 \\
\hline \multicolumn{9}{|l|}{ Type of mental problem/disorder ${ }^{\mathrm{a}}$} \\
\hline $\begin{array}{l}\text { Any current affective } \\
\text { problem/disorder }\end{array}$ & 1.577 & 0.256 & 37.947 & 1 & $<0.001$ & 4.841 & 2.913 & 7.995 \\
\hline $\begin{array}{l}\text { Any current anxiety } \\
\text { problem/disorder }\end{array}$ & 0.327 & 0.265 & 1.974 & 1 & 0.160 & 1.450 & 0.863 & 2.436 \\
\hline $\begin{array}{l}\text { Any current eating } \\
\text { problem/disorder }\end{array}$ & 0.280 & 0.449 & 0.389 & 1 & 0.533 & 1.323 & 0.549 & 3.192 \\
\hline $\begin{array}{l}\text { Any current somatoform } \\
\text { problem/disorder }\end{array}$ & 0.438 & 0.295 & 2.207 & 1 & 0.137 & 1.550 & 0.868 & 2.764 \\
\hline $\begin{array}{l}\text { A current obsessive- } \\
\text { compulsive problem/disorder }\end{array}$ & 0.712 & 0.367 & 3.750 & 1 & 0.053 & 2.037 & 0.991 & 4.186 \\
\hline $\begin{array}{l}\text { A current post-traumatic } \\
\text { stress problem/disorder }\end{array}$ & 0.178 & 0.281 & 0.402 & 1 & 0.526 & 1.195 & 0.689 & 2.070 \\
\hline
\end{tabular}

stress problem/disorder
a All models were significant with a GoF of $\chi^{2}(1) \geq 3.865, \mathrm{p}<0.049$

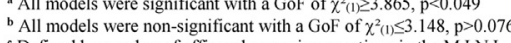

${ }^{\mathrm{C}}$ All models were non-significant with a GoF of $\chi^{2}(1) \leq 3.148, \mathrm{p}>0.076$
Defined by number of affirmed screening questions in the M.I.N.I.

Note: Regressions were calculated using SPSS 24.0 with method "Enter". Significant regressions $(\mathrm{p} \leq 0.05)$ are shaded in grey.

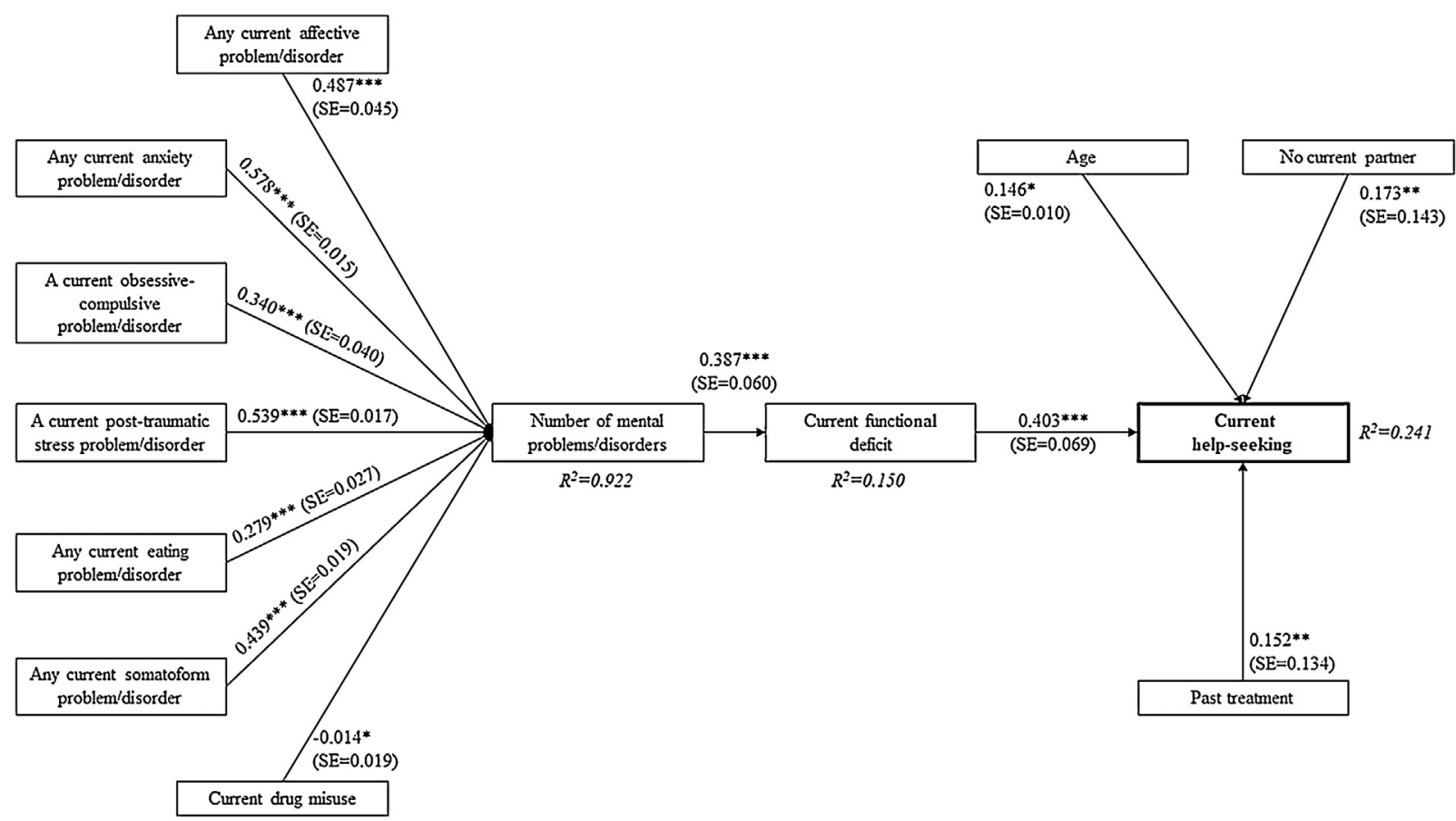

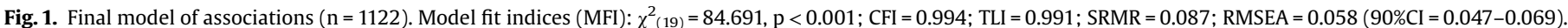

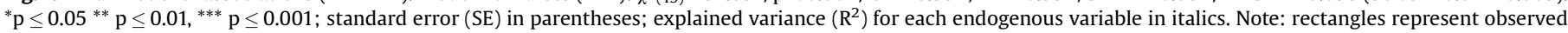
manifest variables; black arrows represent significant regressions. Source: Bern Epidemiological At Risk (BEAR) Study (SNF project number: 135381 ).

frequently hard to treat $[44,45]$. Furthermore, the success of indicated-preventive efforts in terms of a reduction of incidence rates of mental disorders greatly depends on sufficient numbers of persons with early signs of an incipient mental disorder getting into contact with (semi-)professionals for diagnostic clarification and/or treatment. Interestingly, sociodemographic variables, such as age, gender, education, employment status, partnership status, and positive family history of mental disorders, which were often described as important predictors of help-seeking [15,20,21], only had a minor if any effect on current help-seeking within an age range in that non-developmental and non-degenerative mental disorders likely occur first, i.e. between 16 and 40 years of age [25].

We found slightly lower but generally similar prevalence rates and distribution of current mental disorders to those reported for Switzerland in 2010 for a wider age and time span [7]. Consistent with previous reports [8,15], a 'treatment gap' for mental problems/disorders was significant. Overall, $7 \%$ of individuals with 
any current axis-I problem/disorder were currently in any helpseeking contact for their mental problems; in persons with a current mental disorder, the rate was higher but still as low as $14 \%$. When problems/disorders were considered differentially, the rate of current non-help-seekers exceeded $75 \%$ for any diagnostic category. For the frequently reported depressive problems/ disorders (major depression or dysthymia), which have been associated with the highest burden worldwide [1,11,46], the reported rate of any current help-seeking at almost $20 \%$ was comparably high $[23,47]$. Generalized anxiety problems/disorder and rare body dysmorphic problems/disorder were associated with current help-seeking to a slightly higher rate. Previous reports support our finding that depressed mood and anxiousness are main reasons for help-seeking $[23,48]$. Yet, the rate of current treatment for other anxiety problems/disorders was much lower, with specific phobic problems/disorder being the most frequent but least treated. Our study's high prevalence of current specific phobia (6.5\%) is consistent with other epidemiological studies on adults [49-51]. Although specific phobias tend to have an onset in childhood and to frequently remit into adulthood, recent epidemiological studies reported them as a highly disabling, frequently co-morbid condition in adults warranting therapeutic attention [49] as well as a precursor or even predictor of other mental disorders and, thus, a potential target of prevention especially when persisting into late adolescence/early adulthood [51-53]. Thus, we had included them into our analyses as a condition likely warranting clinical attention.

\subsection{Moderators of current help-seeking}

Regarding potential moderators of current help-seeking, we found greater odds for help-seeking in people with functional impairment, higher number of axis-I problems/disorders, current drug misuse, an affective problem/disorder and without partner. The greatest odds for current help-seeking were found for functional impairment and presence of an affective problem/ disorder.

Other sociodemographic variables, specifically younger age or male gender, that were described previously as associated with less help-seeking $[8,54,55]$ were unrelated to current help-seeking in our study. Yet, regarding age, other studies had commonly included individuals up to 65 years old and above [15]; thus, our sample constituted the 'younger age' group for which less helpseeking was reported [21]. Considering this, the missing age effect is not surprising.

\subsubsection{Gender}

The missing systematic gender effect, however, cannot be explained by sampling effects. Although, consistent with their reported higher willingness to seek help [54,55], women reported a higher rate of (past) help-seeking than men, men were currently seeking help more often than women. In studies examining helpseeking irrespective of the presence of mental problems/disorders, the reported higher rate of help-seeking women was assumed to be attributed to their higher lifetime prevalence of mental disorders rather than their general higher willingness to seek help [23]. This assumption was supported by our data showing a decreasing gender effect on help-seeking with increasing severity of mental problems. Thereby, the lowest overall effect that could not be contributed to any specific subgroup was found for helpseeking in participants meeting the criteria of any current mental disorder. This effect may be attributed to an increasing likelihood to seek help and receive longer-term treatment from a mental health professional in individuals with a mental disorder compared with those with only subthreshold mental problems. In a study of persons with anxiety or mood disorders [56], no gender effect showed when only help-seeking from mental health professionals was considered, whereas women dominated when any help-seeking and help-seeking from medical doctors or informal individuals were examined. This interaction between gender and point of help-seeking contact should be explored further in future studies to unveil the gender-specific barriers to help-seeking from or referral to mental health professionals.

Certain indications towards gender-specific barriers were shown in the sensitivity analyses of gender, albeit the lesser and partly poor model fit of the gender-specific models compared to the whole-sample model. Gender differences in the role of age, partnership, and past treatment were found and might be worth considering in future studies. The sensitivity analyses indicated that older age and being single might increase likelihood for helpseeking in women, while this was exclusively increased by past treatment for mental problems in men. However, no gender differences for the more influential clinical variables became apparent. Thus, future information campaigns promoting helpseeking may specifically focus on young females with a partner and males in general.

\subsubsection{Past treatment}

Interestingly, treatment satisfaction did not seem to influence current help-seeking. Considering the reported significant role of attitudes towards help-seeking [57-59], the insignificant role of treatment satisfaction that might shape current attitudes towards help-seeking was surprising.

\subsubsection{Functioning}

Clinical variables, specifically the number of mental problems/ disorders and functional impairment, played the main role in helpseeking, in which the role of mental problems/disorders was mediated by functional deficits. This is in line with previous studies reporting that the co-occurrence of more than one mental disorder, which increased disease burden, was associated with a higher likelihood of using health services [15,60-62] and that veterans commonly do not seek help until problems are severe enough to cause problems in occupational and psychosocial functioning [63]. If the role of functional impairments reflects higher disease burden in the affected persons and/or in their significant others who, in the presence of observable functional changes and symptoms, might increasingly encourage help-seeking should be studied in the future. A recent study on help-seeking for psychoses indicated that the role of others in initiating help-seeking becomes more significant with the increasing severity of the symptoms [64]. If this holds true for other disorders, strategies to promote helpseeking should focus particularly on persons who already have a mild current axis-I disorder or some persistent mental problems but no functional deficit and who are not currently help-seeking in order to prevent chronicity and/or exacerbation of symptoms, and functional decline.

\subsection{Strengths and limitations}

Next to the strengths of our study, such as the large sample size, clinician-assessment, and the consideration of not only mental problems/disorders but also psychosocial functioning, the restricted age range (16-40 years) limits the wider generalizability. The cross-sectional design of our study is certainly another limitation that it shares with many other epidemiological studies and that prevented us from examining causal associations, and that might have led to an overestimation of the 'treatment gap' in persons with spontaneous remission of mental problems/disorders. Yet another limitation that our study shares with other epidemiological studies is the lack of a physical examination to rule out somatic causes of the mental problems/disorders, although we tried to 
minimize this risk by asking for known neurological disorders or other acute or chronic conditions that might influence the current mental state. Moreover, the complex pathway to early helpseeking may involve additional factors, such as attitudinal barriers [58], a low perceived need for treatment [65], low mental health literacy [66,67], or organizational problems, such as job obligations [68].

\subsection{Conclusion}

Overall, our results document a lack of early help-seeking for mental disorders that seems to occur worldwide and largely resistant to change. For example, between 2005 and 2010, neither the 12 -month prevalence of mental disorders (38\%) nor the rate of adequate treatment utilization and provision for mental disorders ( $26 \%$ of all cases with mental disorders) had changed substantially in Europe [1]. In our study, the point-prevalence rates of $13.2 \%$ for mental disorders and of $13.6 \%$ for any help-seeking do not give a more optimistic view for Switzerland. Despite its excellent Healthcare Access and Quality Index of 92\% [69] and its top-10 position in gross domestic product per capita [70], Switzerland registered an increase in the number of young adults and persons with low income who avoid or delay medical help-seeking for financial reasons from 10\% in 2010 to $23 \%$ in 2016 [71]. It must be assumed that this trend might be specifically pronounced in helpseeking for mental problems that are commonly highly related to fear of stigmatization/discrimination, as well as to low perceived need for help and a strong desire to handle the problem on one's own [58,65-67]. Yet, to fight the increasing burden and costs of mental disorders and to decrease incidence of severe mental disorders [1,7], it will be crucial to thoroughly understand barriers to help-seeking in order to successfully establish preventive measures on individual and societal levels in concerted action involving researchers, policymakers, and health specialists $[72,73]$.

\section{Conflict of interest}

Drs Michel, Schnyder, Schmidt, Schultze-Lutter, and MSc Groth have declared that there are no conflicts of interest in relation to the subject of this study.

Dr Schimmelmann has been a consultant and/or advisor to or has received honoraria from AstraZeneca, Bristol-Myers Squibb, Eli Lilly, Janssen, Novartis, and Shire.

\section{Funding}

This research was supported by grant 32003B_135381 from the Swiss National Science Foundation (Bern, Switzerland). The supporter had no role in the design, analysis, interpretation, or publication of this study.

\section{Acknowledgement}

None.

\section{Appendix A. Supplementary data}

Supplementary material related to this article can be found, in the online version, at doi:https://doi.org/10.1016/j.eurpsy.2018.06.009.

\section{References}

[1] Wittchen H.U., Jacobi F, Rehm J, Gustavsson A, Svensson M, Jönsson B, Olesen J, Allgulander C, Alonso J, Faravelli C, Fratiglioni L, Jennum P, Lieb R, Maercker A, van Os J, Preisig M, Salvador-Carulla L, Simon R, Steinhausen HC. The size and burden of mental disorders and other disorders of the brain in Europe 2010. Eur Neuropsychopharmacol 2011;21(9):655-79.
[2] Jones PB. Adult mental health disorders and their age at onset. Br J Psychiatry Suppl 2013;54:5-10.

[3] Vaingankar JA, Rekhi G, Subramaniam M, Abdin E, Chong SA. Age of onset of life-time mental disorders and treatment contact. Soc Psychiatry Psychiatr Epidemiol 2013;48(5):835-53.

[4] Kessler RC, Amminger GP, Aguilar-Gaxiola S, Alonso J, Lee S, Ustün TB. Age of onset of mental disorders: a review of recent literature. Curr Opin Psychiatry 2007;20(4):359-64.

[5] Gore FM, Bloem PJ, Patton GC, Ferguson J, Joseph V, Coffey C, Sawyer SM, Mathers CD. Global burden of disease in young people aged 10-24 years: a systematic analysis. Lancet 2011;377(9783):2093-102.

[6] Olesen J, Gustavsson A, Svensson M, Wittchen HU, Jönsson B, CDBE2010 study group, European Brain Council. The economic cost of brain disorders in Europe. Eur J Neurol 2012;19(1):155-62.

[7] Gustavsson A, Svensson M, Jacobi F, Allgulander C, Alonso J, Beghi E, Dodel R, Ekman M, Faravelli C, Fratiglioni L, Gannon B, Jones DH, Jennum P, Jordanova A, Jönsson L, Karampampa K, Knapp M, Kobelt G, Kurth T, Lieb R, Linde M, Ljungcrantz C, Maercker A, Melin B, Moscarelli M, Musayev A, Norwood F, Preisig M, Pugliatti M, Rehm J, Salvador-Carulla L, Schlehofer B, Simon R, Steinhausen HC, Stovner LJ, Vallat JM, Van den Bergh P, van Os J, Vos P, Xu W, Wittchen HU, Jönsson B, Olesen J, CDBE2010Study Group. Cost of disorders of the brain in Europe 2010. Eur Neuropsychopharmacol 2011;21(10):718-79.

[8] Wang PS, Angermeyer M, Borges G, Bruffaerts R, Tat Chiu W, DE Girolamo G, Fayyad J, Gureje O, Haro JM, Huang Y, Kessler RC, Kovess V, Levinson D, Nakane Y, Oakley Brown MA, Ormel JH, Posada-Villa J, Aguilar-Gaxiola S, Alonso J, Lee S, Heeringa S, Pennell BE, Chatterji S, Ustün TB. Delay and failure in treatment seeking after first onset of mental disorders in the World Health Organization's World Mental Health Survey Initiative. World Psychiatry 2007;6(3):177-85.

[9] World Health Organization (WHO). Prevention of mental disorders: effective interventions and policy options: summary report. Geneva, Switzerland: WHO; 2004.

[10] Olfson M, Marcus SC, Druss B, Elinson L, Tanielian T, Pincus HA. National trends in outpatient treatment of depression. JAMA 2002;287(2):203-9.

[11] Thornicroft G, Chatterji S, Evans-Lacko S, Gruber M, Sampson N, AguilarGaxiola S, Al-Hamzawi A, Alonso J, Andrade L, Borges G, Bruffaerts R, Bunting B, de Almeida JM, Florescu S, de Girolamo G, Gureje O, Haro JM, He Y, Hinkov H, Karam E, Kawakami N, Lee S, Navarro-Mateu F, Piazza M, Posada-Villa J, de Galvis YT, Kessler RC. Undertreatment of people with major depressive disorder in 21 countries. Br J Psychiatry 2017;210(2):119-24.

[12] Wang PS, Demler O, Olfson M, Pincus HA, Wells KB, Kessler RC. Changing profiles of service sectors used for mental health care in the United States. Am J Psychiatry 2006;163(7):1187-98.

[13] Demyttenaere K, Bruffaerts R, Posada-Villa J. Prevalence, severity, and unmet need for treatment of mental disorders in the World Health Organization World Mental Health surveys. JAMA 2004:291(21):2581-90.

[14] Patel V, Maj M, Flisher AJ, De Silva MJ, Koschorke M, Prince M, WPA Zonal and Member Society Representatives. Reducing the treatment gap for mental disorders: a WPA survey. World Psychiatry 2010;9(3):169-76.

[15] Bijl RV, Ravelli A. Psychiatric morbidity, service use, and need for care in the general population: results of The Netherlands Mental Health Survey and Incidence Study. Am J Public Health 2000;90(4):602-7.

[16] Harris MG, Hobbs MJ, Burgess PM, Pirkis JE, Diminic S, Siskind DJ, Andrews G Whiteford HA. Frequency and quality of mental health treatment for affective and anxiety disorders among Australian adults. Med J Aust 2015;202(4):185-9.

[17] Kessler RC, Brown RL, Broman CL. Sex differences in psychiatric help-seeking: evidence from four-large-scale surveys. J Health Soc Behav 1982;22(1):49-64.

[18] Ladwig KH, Marten-Mittag B, Formanek B, Dammann G. Gender differences of symptom reporting and medical health care utilization in the German population. Eur J Epidemiol 2000;16(6):511-8.

[19] Levinson D, Ifrah A. The robustness of the gender effect on help-seeking for mental health need in three subcultures in Israel. Soc Psychiatry Psychiatr Epidemiol 2010;45(3):337-44.

[20] Oliver MI, Pearson N, Coe N, Gunnell D. Help-seeking behaviour in men and women with common mental health problems: cross-sectional study. $\mathrm{Br} \mathrm{J}$ Psychiatry 2005;186:297-301.

[21] Roskar S, Bracic MF, Kolar U, Lekic K, Juricic NK, Grum AT, Dobnik B, Postuvan V, Vatovec M. Attitudes within the general population towards seeking professional help in cases of mental distress. Int J Soc Psychiatry 2017;63 (7):614-21.

[22] Mackenzie CS, Reynolds K, Cairney J, Streiner DL, Sareen J. Disorderspecific mental health service use for mood and anxiety disorders: associations with age, sex, and psychiatric comorbidity. Depress Anxiety 2012;29(3):234-42.

[23] Alonso J, Angermeyer MC, Bernert S, Bruffaerts R, Brugha TS, Bryson H, de Girolamo G, Graaf R, Demyttenaere K, Gasquet I, Haro JM, Katz SJ, Kessler RC, Kovess V, Lépine JP, Ormel J, Polidori G, Russo LJ, Vilagut G, Almansa J, Arbabzadeh-Bouchez S, Autonell J, Bernal M, Buist-Bouwman MA, Codony M, Domingo-Salvany A, Ferrer M, Joo SS, Martínez-Alonso M, Matschinger $\mathrm{H}$, Mazzi F, Morgan Z, Morosini P, Palacín C, Romera B, Taub N, Vollebergh WA, ESEMeD/MHEDEA 2000 investigators, European Study of the Epidemiology of Mental Disorders (ESEMeD) project. Use of mental health services in Europe: results from the European Study of the Epidemiology of Mental Disorders (ESEMeD) project. Acta Psychiatr Scand Suppl 2004;(420):47-54.

[24] Schultze-Lutter F, Michel C, Ruhrmann S, Schimmelmann BG. Prevalence and clinical relevance of interview-assessed psychosis risk symptoms in the young adult community. Psychol Med 2018;48(7):1167-78. 
[25] Michel C, Schimmelmann BG, Schultze-Lutter F. Demographic and clinical characteristics of diagnosed and non-diagnosed psychotic disorders in the community. Early Interv Psychiatry 2018;12(1):87-90.

[26] Sheehan DV, Lecrubier Y, Sheehan KH, Amorim P, Janavs J, Weiller E, Herguet T, Baker R, Dunbar GC. The Mini-International Neuropsychiatric Interview (M.I N.I.): the development and validation of a structured diagnostic psychiatric interview for DSM-IV and ICD-10. J Clin Psychiatry 1998;59(Suppl. 20):22-33.

[27] Wang J, Adair CE, Patten SB. Mental health and related disability among workers: a population-based study. Am J Ind Med 2006;49(7):514-22.

[28] Sheehan DV, Lecrubier Y, Sheehan KH, Janavs J, Weiller E, Keskiner A, Schinka J, Knapp E, Sheehan MF, Dunbar GC. The validity of the Mini International Neuropsychiatric Interview (Mini) according to the SCID-P and its reliability. Eur Psychiatry 1997;12(5):232-41.

[29] Rohde P, Lewinsohn PM, Seeley JR. Comparability of telephone and face-toface interviews in assessing axis I and II disorders. Am J Psychiatry 1997;154 (11):1593-8.

[30] New York State Office of Alcoholism and Substance abuse Services (OASAS) Screening for co-occurring disorders user guide for the Modified Mini Screen (MMS): adapted for New York City Department of Mental Health and Menta Hygiene Quality Impact Initiative. Albany, USA: OASAS; 2006.

[31] Alexander MJ, Haugland G, Lin SP, Bertollo DN, McCorry FA. Mental health screening in addiction, corrections and social service settings: validating the MMS. Int J Ment Health Addict 2008;6(1):105-19.

[32] Gater R, de Almeida e Sousa B, Barrientos G, Caraveo J, Chandrashekar CR, Dhadphale M, Goldberg D, al Kathiri AH, Mubbashar M, Silhan K, Thong D, Torres-Gonzales F, Satorius N. The pathways to psychiatric care: a crosscultural study. Psychol Med 1991;21(3):761-74.

[33] Gater R, Jordanova V, Maric N, Alikaj V, Bajs M, Cavic T, Dimitrov H, Iosub D, Mihai A, Szalontay AS, Helmchen H, Sartorius N. Pathways to psychiatric care in Eastern Europe. Br J Psychiatry 2005;186:529-35.

[34] Bhui K, Ullrich S, Coid JW. Which pathways to psychiatric care lead to earlier treatment and a shorter duration of first-episode psychosis? BMC Psychiatry 2014; $14: 72$.

[35] Büssing A, Fischer J, Haller A, Heusser P, Ostermann T, Matthiessen PF. Validation of the brief multidimensional life satisfaction scale in patients with chronic diseases. Eur J Med Res 2009;14(4):171-7.

[36] American Psychiatric Association (APA). Diagnostic and statistical manual of mental disorders. fourth ed. Washington, USA: APA; 1994.

[37] Rybarczyk B. Social and occupational functioning assessment scale [SOFAS]. In: Kreutzer J, Deluca J, Caplan LXIII B, editors. Encyclopedia of clinical neuropsychology, vol. 1. New York: Springer Reference; 2011 p. 2313.

[38] Hilsenroth MJ, Ackerman SJ, Blagys MD, Baumann BD, Baity MR, Smith SR, Price JL, Smith CL, Heindselman TL, Mount MK, Holdwick Jr. DJ. Reliability and validity of DSM-IV axis V. Am J Psychiatry 2000;157(11):1858-63.

[39] Morosini PL, Magliano L, Brambilla L, Ugolini S, Pioli R. Development, reliability and acceptability of a new version of the DSM-IV Social and Occupational Functioning Assessment Scale (SOFAS) to assess routine social functioning. Acta Psychiatr Scand 2000;101(4):323-9.

[40] Schimmelmann BG, Michel C, Martz-Irngartinger A, Linder C, Schultze-Lutter F. Age matters in the prevalence and clinical significance of ultra-high-risk for psychosis symptoms and criteria in the general population: findings from the BEAR and BEARS-kid studies. World Psychiatry 2015;14(2):189-97.

[41] Brown TA. Confirmatory factor analysis for applied researchers. New York USA: Guilford Press; 2006.

[42] Muthén BO. Goodness of fit with categorical and other nonnormal variables. In: Bollen KA, Long JS, editors. Testing structural equation models. Newbury Park, CA, USA: Sage; 1993. p. 205-34.

[43] Rosseel Y. lavaan: an R package for structural equation modeling. J Stat Softw 2012;48:1-36. . URL http://www.jstatsoft.org/v48/i02/.

[44] Whiteford HA, Degenhardt L, Rehm J, Baxter AJ, Ferrari AJ, Erskine HE, Charlson FJ, Norman RE, Flaxman AD, Johns N, Burstein R, Murray CJ, Vos T. Global burden of disease attributable to mental and substance use disorders: finding from the Global Burden of Disease Study. Lancet 2010;382(9904):1575-86.

[45] Campion J, Bhui K, Bhugra D, European Psychiatric Association. European Psychiatric Association (EPA) guidance on prevention of mental disorders. Eur Psychiatry 2012;27(2):68-80.

[46] Schultze-Lutter F, Michel C, Schmidt SJ, Schimmelmann BG, Maric NP, Salokangas RKR, Riecher-Rössler A, van der Gaag M, Nordentoft M, Raballo A, Meneghelli A, Marshall M, Morrison A, Ruhrmann S, Klosterkötter J. EPA guidance on the early detection of clinical high risk states of psychoses. Eur Psychiatry 2015;30:405-16.

[47] Wittchen HU, Jacobi F. Size and burden of mental disorders in Europe-a critical review and appraisal of 27 studies. Eur Neuropsychopharmacol 2005;15 (4):357-76.

[48] Schultze-Lutter F, Michel C, Ruhrmann S, Schimmelmann BG. Prevalence and clinical significance of DSM-5-attenuated psychosis syndrome in adolescents and young adults in the general population: the Bern Epidemiological At-Risk (BEAR) study. Schizophr Bull 2014:40(6):1499-508.

[49] Stinson FS, Dawson DA, Patricia Chou S, Smith S, Goldstein RB, June Ruan W, Grant BF. The epidemiology of DSM-IV specific phobia in the USA: results from the National Epidemiologic Survey on alcohol and related conditions. Psychol Med 2007;37(7):1047-59.
[50] Bandelow B, Michaelis S. Epidemiology of anxiety disorders in the 21st century. Dialogues Clin Neurosci 2015;17(3):327-35.

[51] Wardenaar KJ, Lim CCW, Al-Hamzawi AO, Alonso J, Andrade LH, Benjet C, Bunting B, de Girolamo G, Demyttenaere K, Florescu SE, Gureje O, Hisateru T, Hu C, Huang Y, Karam E, Kiejna A, Lepine JP, Navarro-Mateu F, Oakley Browne M, Piazza M, Posada-Villa J, Ten Have ML, Torres Y, Xavier M, Zarkov Z, Kessler RC, Scott KM, de Jonge P. The cross-national epidemiology of specific phobia in the World Mental Health Surveys. Psychol Med 2017;47(10):1744-60.

[52] Albor YC, Benjet C, Méndez E, Medina-Mora ME. Persistence of specific phobia from adolescence to early adulthood: longitudinal follow-up of the Mexican adolescent mental health survey. J Clin Psychiatry 2017;78(3):340-6.

[53] Lieb R, Miché M, Gloster AT, Beesdo-Baum K, Meyer AH, Wittchen HU. Impact of specific phobia on the risk of onset of mental disorders: a 10-year prospective-longitudfinal community study of adolescent and young adults. Depress Anxiety 2016;33(7):667-75.

[54] Dey M, Jorm AF. Social determinants of mental health service utilization in Switzerland. Int J Public Health 2017;62(1):85-93.

[55] Mackenzie CS, Gekoski WL, Knox VJ. Age, gender, and the underutilization of mental health services: the influence of help-seeking attitudes. Aging Mental Health 2006;10(6):574-82.

[56] Susukida R, Mojtabai R, Mendelson T. Sex differences in help seeking for mood and anxiety disorders in the national comorbidity survey-replication. Depress Anxiety 2015;32(11):853-60.

[57] Mojtabai R, Evans-Lacko S, Schomerus G, Thornicroft G. Attitudes toward mental health help seeking as predictors of future help-seeking behavior and use of mental health treatments. Psychiatr Serv 2016;67(6):650-7.

[58] Schnyder N, Panczak R, Groth N, Schultze-Lutter F. Association between mental-health related stigma and active help-seeking: systematic review and meta-analysis. Br J Psychiatry 2017;210(4):261-8.

[59] Schnyder N, Michel C, Panczak R, Ochsenbein S, Schimmelmann BG, SchultzeLutter F. The interplay of etiological knowledge and mental illness stigma on healthcare utilisation in the community: a structural equation model. Eur Psychiatry 2018;51:48-56.

[60] Andrews G, Henderson S, Hall W. Prevalence, comorbidity, disability and service utilisation. Overview of the Australian National Mental Health Survey. Br J Psychiatry 2001;178:145-53.

[61] Katz SJ, Kessler RC, Frank RG, Leaf P, Lin E, Edlund M. The use of outpatient mental health services in the United States and Ontario: the impact of mental morbidity and perceived need for care. Am J Public Health 1997;87(7):113643.

[62] Jacobi F, Höfler M, Strehle J, Mack S, Gerschler A, Scholl L, Busch MA, Maske U, Hapke U, Gaebel W, Maier W, Wagner M, Zielasek J, Wittchen HU. Mental disorders in the general population: study on the health of adults in Germany and the additional module mental health (DEGS1-MH). Nervenarzt 2014;85:77-87.

[63] Hines LA, Goodwin L, Jones M, Hull L, Wessely S, Fear NT, Rona RJ. Factors affecting help seeking for mental health problems after deployment to Iraq and Afghanistan. Psychiatric Serv 2014;65(1):98-105.

[64] Schultze-Lutter F, Rahman J, Ruhrmann S, Michel C, Schimmelmann BG, Maier W, Klosterkötter J. Duration of unspecific prodromal and clinical high risk states, and early help-seeking in first-admission psychosis patients. Soc Psychiatry Psychiatr Epidemiol 2015;50(12):1831-41.

[65] Andrade LH, Alonso J, Mneimneh Z, Wells JE, Al-Hamzawi A, Borges G, Bromet E, Bruffaerts R, de Girolamo G, de Graaf R, Florescu S, Gureje O, Hinkov HR, Hu C, Huang Y, Hwang I, Jin R, Karam EG, Kovess-Masfety V, Levinson D, Matschinger H, O’Neill S, Posada-Villa J, Sagar R, Sampson NA, Sasu C, Stein DJ, Takeshima T, Viana MC, Xavier M, Kessler RC. Barriers to mental health treatment: results from the WHO World Mental Health surveys. Psychol Med 2014;44(6):1303-17.

[66] Jorm AF. Mental health literacy: empowering the community to take action for better mental health. Am Psychol 2012;67(3):231-43.

[67] Kelly CM, Jorm AF, Wright A. Improving mental health literacy as a strategy to facilitate early intervention for mental disorders. Med J Aust 2007;187(7 Suppl):26-30.

[68] Lo CC, Cheng TC. The role of social structural factors in treatment of mental health disorder. J Ment Health 2012;21(5):430-8.

[69] GBD 2015 Healthcare Access and Quality Collaborators. Healthcare Access and Quality Index based on mortality from causes amenable to personal health care in 195 countries and territories, 1990-2015: a novel analysis from the Global Burden of Disease Study 2015. Lancet 2017;390(10091):231-66.

[70] Wikipedia. List of countries by GDP (PPP) per capita. (Accessed 17 May 2018). https://simple.wikipedia.org/wiki/List_of_countries_by_GDP_(PPP) _per_capita.

[71] Osborn R, Squires D, Doty MM, Sarnak DO, Schneider EC. In new survey of eleven countries, US adults still struggle with access to and affordability of health care. Health Aff 2016;35(12):2327-36.

[72] Grépin KA, Reich MR. Conceptualizing integration: a framework for analysis applied to neglected tropical disease control partnerships. PLoS Negl Trop Dis 2007;2(4):e174.

[73] De Cock KM, El-Sad WM, Ghebreyesus TA. Game changers: why did the scaleup of HIV treatment work despite weak health systems? J Acquir Immune Defic Syndr 2011;57(Suppl. 2):61-3. 